\title{
Vascular anatomy of the thenar eminence: its relevance to a pedicled or free thenar flap
}

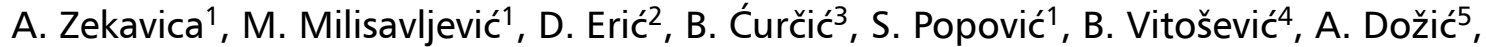 \\ B.V. Štimec ${ }^{6}$, R. Manojlović ${ }^{7}$ \\ ${ }^{1}$ Laboratory for Vascular Anatomy, Institute of Anatomy, Faculty of Medicine, University of Belgrade, Belgrade, Serbia \\ ${ }^{2}$ Department of Plastic, Reconstructive and Hand Surgery, Faculty of Medicine, University of East Sarajevo, \\ Foča, Republic of Srpska, Bosnia and Herzegovina \\ 3 Institute of Anatomy, Faculty of Medicine, University of East Sarajevo, Foča, Republic of Srpska, Bosnia and Herzegovina \\ ${ }^{4}$ Medical Department, Faculty of Sport and Physical Education, University of K. Mitrovica, K. Mitrovica, Serbia \\ 5 Institute of Anatomy, Faculty of Dentistry, University of Belgrade, Belgrade, Serbia \\ ${ }^{6}$ Faculty of Medicine, Department of Cellular Physiology and Metabolism, Anatomy Sector, University of Geneva, \\ Geneva, Switzerland \\ 7Institute for Orthopaedic Surgery and Traumatology, Clinical Centre of Serbia, Faculty of Medicine, \\ University of Belgrade, Belgrade, Serbia
}

[Received 13 October 2016; Accepted: 12 November 2016]

Background: The aim of this study was to clarify the arterial supply of the skin covering the prominent part of the thenar eminence in order to describe the possibility and potential for harvesting a pedicled or a free flap from the thenar eminence.

Materials and methods: The arteries were studied in 30 post-mortem specimens of human hands; 3 previously perfused with $4 \%$ formaldehyde solution, and injected with black India ink, and 27 injected with methyl-methacrylate and afterwards corroded in 40\% potassium hydroxide solution.

Results: In all hands we found two little palmar arteries coming from the anatomical snuff-box portion of the radial artery. We labelled the first (proximal) branch as the middle thenar artery, because it supplies the middle third of the thenar eminence skin. Its diameter varied from 0.25 to $0.55 \mathrm{~mm}$ (mean $0.4 \mathrm{~mm}$ ). The distal, more prominent branch of the radial artery, vascularised the lateral third of the thenar eminence skin, and was named the lateral thenar artery; its diameter ranged from 0.40 to $0.90 \mathrm{~mm}$ (mean $0.67 \mathrm{~mm}$ ). The superficial palmar branch of the radial artery, always present, was classified as: hypoplastic, average or prominent, with a diameter ranging from 0.8 to $2.7 \mathrm{~mm}$ (mean $1.47 \mathrm{~mm}$ ). Conclusions: Three individually developed branches of the radial artery supplied the skin of the thenar eminence. Cutaneous branches of these three arteries were interconnected via anastomotic vessels. (Folia Morphol 2017; 76, 2: 232-238)

Key words: lateral thenar artery, middle thenar artery, superficial palmar branch, thenar eminence

\section{INTRODUCTION}

Many authors and standard anatomical textbooks have dealt with the vasculature of the hand $[3,11$, $12,15,17]$. Only a few articles have been devoted to the cutaneous blood supply of the thenar eminence itself $[4,13,16]$, and there are only scarce data available on the clinical importance of the collateral branches of the radial artery (RA) within the 


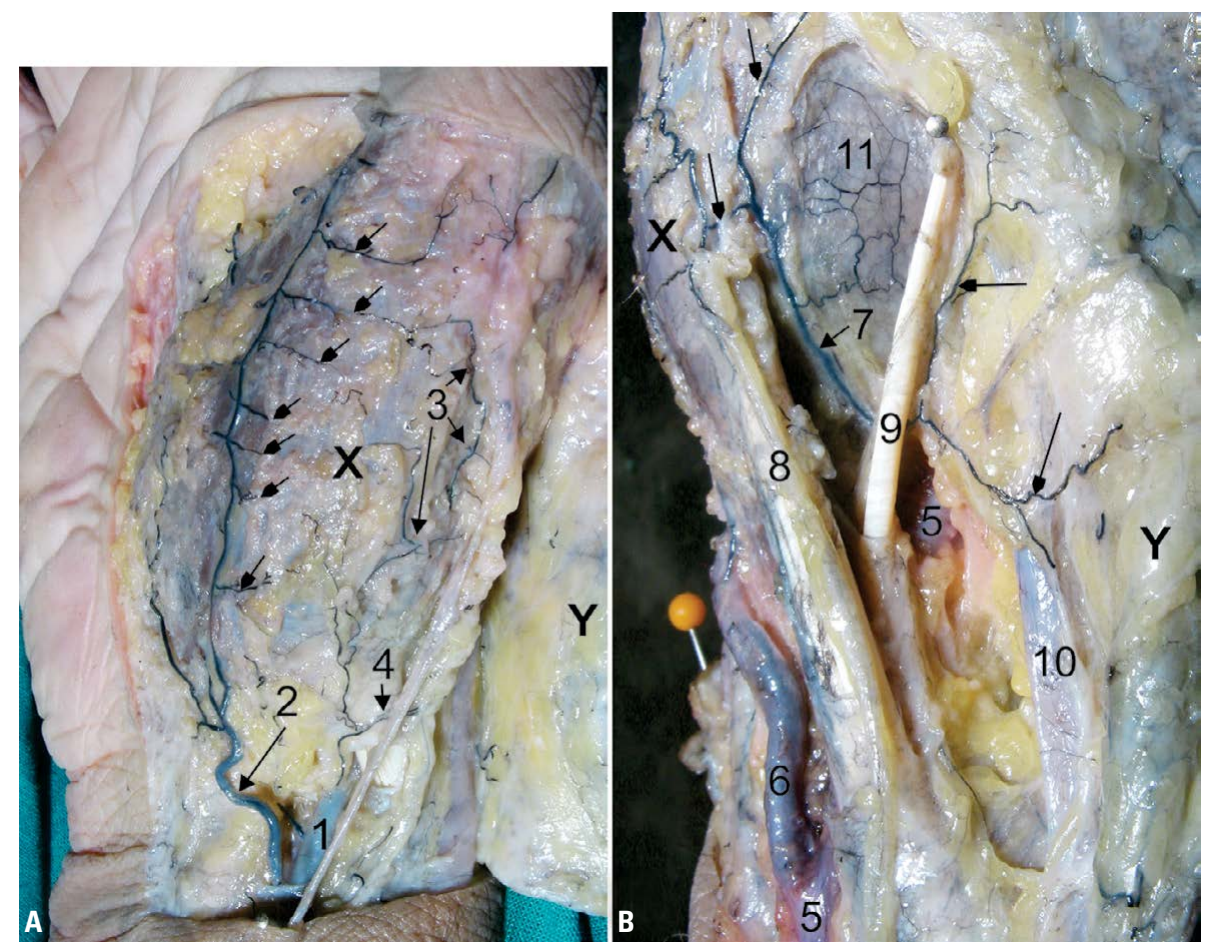

Figure 1. Dissections of the right thenar eminence $(\mathrm{X})$ after India ink and gelatine injection and elevation of the skin flap (Y); A. Palmar view; the radial artery (1) sends hypoplastic the superficial palmar branch (SPB) (2) with transverse anastomotic subcutaneous arteries (arrows) with the lateral thenar artery (LTA) (3), and the middle thenar artery (MTA) (4) branches. B. Lateral view; the radial artery (5), after giving off a well-developed SPB (6), enters the anatomical snuff-box and sends the LTA (7) along the lateral border of the thenar eminence; 8 - abductor pollicis longus tendon; 9 - extensor pollicis brevis tendon; 10 - extensor pollicis longus tendon; 11 - osteoperiosteal branches at the level of the base of the first metacarpal bone; arrows - subcutaneous branches of the LTA.

anatomical snuff-box $[7,9,18]$. To our knowledge, there are only 3 articles in the literature which partly address the microanatomy of the RA branches in this region $[1,2,14]$.

We still do not know the precise arterial vascular pattern of the thenar eminence, important for plastic and reconstructive hand surgery. The conversance of thenar eminence vascularisation is the basis for the design and harvesting the flaps for reconstruction in numerous cases: palmar skin defects, loss of fingertip pulp with tendon and bone, in nerve exposure, or in case of a longstanding scaphoid bone non-union $[2,5$, 17]. Therefore, insufficiency of relevant anatomic data and great surgical significance of this small area call for a study with scientific and practical implications.

The aim of our detailed study concerning the arteries in this region is to lay important anatomical basis for improving the safety of harvesting these flaps.

\section{MATERIALS AND METHODS}

For this microanatomical study we used 30 human hands (13 right, 17 left) with the vasculature of the upper limb completely preserved, from the collection of the Laboratory for Vascular Anatomy. They belonged to adults ( 18 males and 12 females), with a mean age of 51.6 years (range 26-62 years). After perfusion of the arterial system with isotonic saline solution, and afterwards with a $4 \%$ formaldehyde solution, a $10 \%$ mixture of India ink and gelatine was injected into the RA, in 3 hands. The hand specimens underwent fixation for 3 weeks, and were then carefully dissected. Twenty-seven hands were used for preparing the vascular casts. Their arterial system was injected with methylmethacrylate. As soon as polymerisation was completed, a $40 \%$ solution of potassium hydroxide was applied for corrosion, and the cast rinsed afterwards. Thenar blood vessels were examined under the stereoscopic microscope, precise sketches of each specimen were made, and measurements of the vessels were accomplished under ocular micrometre. The main advantage of this technique is that it enables a precise 3-dimensional spatial distinction between the vessels, a clear definition of anastomoses, and finally, this method allows 


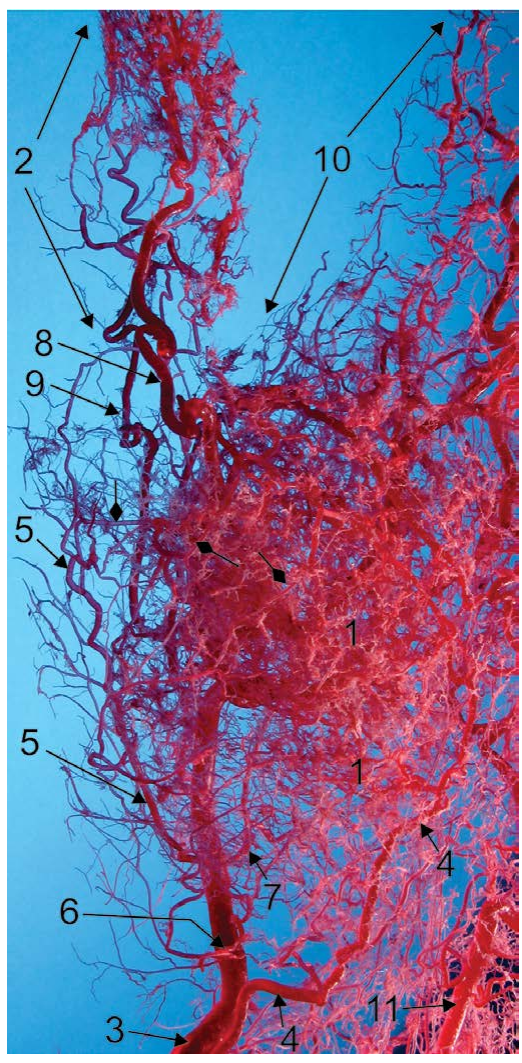

Figure 2. The vascular cast of the left hand presenting arteries of the thenar eminence (1) and the thumb (2). Note the radial artery (3) and its branches: the superficial palmar branch (4), the lateral thenar artery (5), and their anastomotic vessels (arrows), the middle thenar artery (6), the second dorsal metacarpal artery (7), the lateral palmar digital artery of the thumb (8), the medial palmar digital artery of the thumb (9); 10 - index finger; 11 - ulnar artery (ventral view).

each vessel to be followed to its smallest rami, thus permitting a detailed description of its territory of irrigation, much better than any dissection. A drawback of this method is the length of time required to complete the preparation and observe in detail even one single specimen.

\section{RESULTS}

In this study, we explored the participation of the superficial palmar branch (SPB) and the collateral branches of the RA in vascularisation of the skin of the thenar eminence over the abductor pollicis brevis and flexor pollicis brevis muscles. Cutaneous arteries of the distal part of the thenar eminence originated from the lateral palmar digital artery of the thumb. The skin of the thenar eminence was supplied with cutaneous branches which traversed the muscles and the thenar fascia.

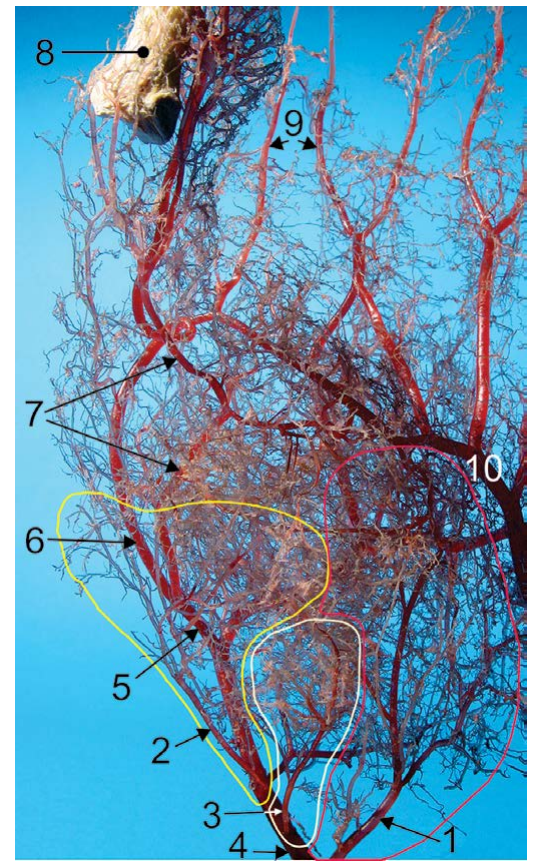

Figure 3. Ventral (palmar) view of the vascular cast of the left hand showing three vascular territories of the thenar eminence skin supplied by the superficial palmar branch $(1$, red), the lateral thenar artery $(2$, yellow), and the middle thenar artery $(3$, white); 4 - radial artery; 5 - princeps pollicis artery; 6 - medial palmar digital artery of the thumb; 7 - lateral palmar digital artery of the thumb; 8 - distal phalanx of the thumb; 9 - index finger palmar digital arteries; 10 - ulnar artery.

After giving rise to the SPB, the RA turned posteriorly across the floor of the anatomical snuff-box (Fig. 1) where, in all specimens $(100 \%)$, it gave off two palmar branches (laterally and ventrally) to the thenar eminence, and one dorsal branch medially, to the dorsal side of the hand.

The two palmar branches were: the middle thenar artery (MTA), and the lateral thenar artery (LTA). The diameter of the MTA was smaller, $0.25-0.55 \mathrm{~mm}$ (mean $0.42 \mathrm{~mm}$ ). This artery plunged into the abductor pollicis brevis muscle, re-emerged from it and supplied the skin of the middle third of the thenar eminence base (Figs. 2-5).

Lateral thenar artery was the more distal branch of the RA. Its diameter, ranging from 0.40 to $0.90 \mathrm{~mm}$ (mean $0.67 \mathrm{~mm}$ ) was larger than of the MTA. Regardless of this difference, the LTA was very dominant in only $25 \%$ of cases when its cutaneous branches accounted for most of the cutaneous territory shared with the MTA.

Lateral thenar artery coursed under the tendon of the extensor pollicis brevis muscle and ran subcutaneously along the lateral border of the abductor 


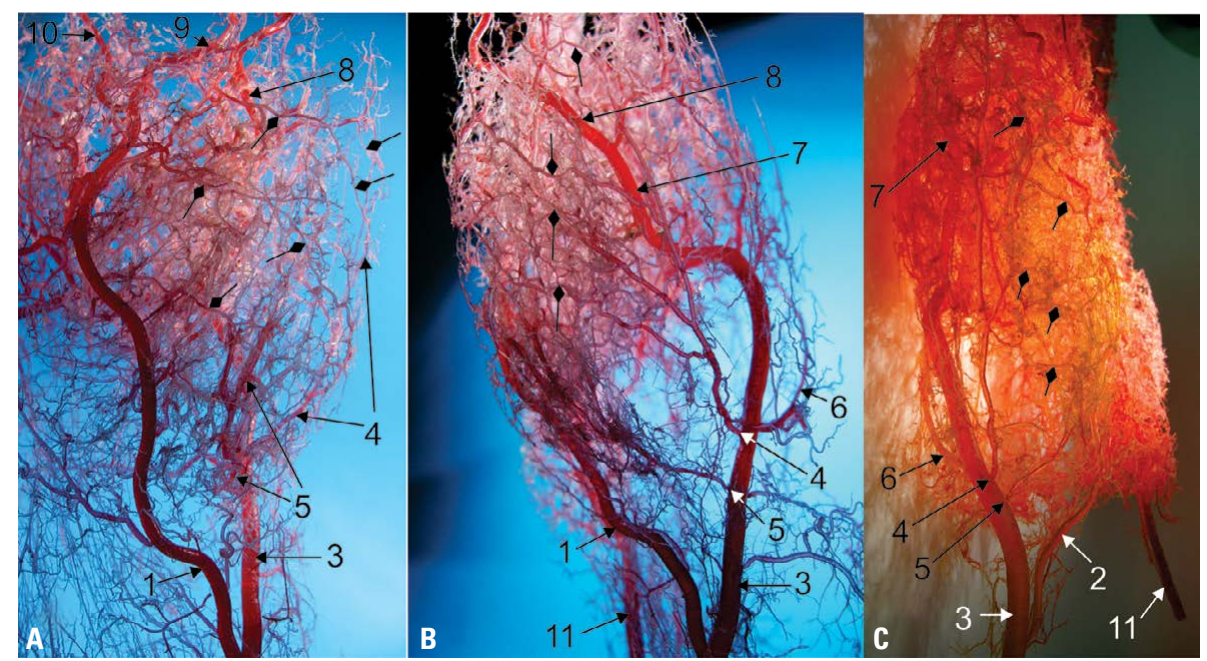

Figure 4. Corrosion casts of the right hand; ventral view (A), and lateral view (B), of the same thenar eminence, and left hand; lateral view of the thenar eminence (C). The prominent superficial palmar branch (SPB) (1) and the average developed SPB (2) of the radial artery (3), and anastomotic subcutaneous vessels (arrows) with the lateral thenar artery (4), and the middle thenar artery (5) branches; 6 - second dorsal metacarpal artery; 7 - princeps pollicis artery; 8 — the lateral palmar digital artery of the thumb; 9 - medial palmar digital artery of the thumb; 10 - radialis indicis artery; 11 - ulnar artery.

pollicis brevis muscle. This artery always followed the lateral border of the thenar eminence to the level of the metacarpophalangeal joint of the thumb. It supplied muscles and skin in the lateral third of the thenar eminence portion. It gave off long and delicate cutaneous branches for the vascularisation of the skin of the lateral part of the thenar eminence (Figs. 1-6).

The second dorsal metacarpal artery was the third branch of the RA's dorsal part within the anatomical snuff-box. Oriented dorsally and medially, larger than the others, it always originated at the level of the LTA, and descended over the second intermetacarpal space (Figs. 2-4).

These were constant arteries, supplying the prominent portion of the thenar eminence skin, and their diameters depended on the radial artery's SPB. In terms of the degree of development, the latter can be classified as prominent, average or hypoplastic. The distance between the points of origins of SPB and MTA on the RA was $17.0 \mathrm{~mm}$, on average (range 13.4-19.9 mm). The average distance from the exit of the MTA to the beginning of the LTA was $5.8 \mathrm{~mm}$ (range 4.3-9.1 mm).

The SPB of the RA was found in all specimens (100\%). This branch originated from the RA before the latter passed into the anatomical snuff-box, at the level of the wrist joint and the styloid process of the radius. The SPB of the RA coursed through the

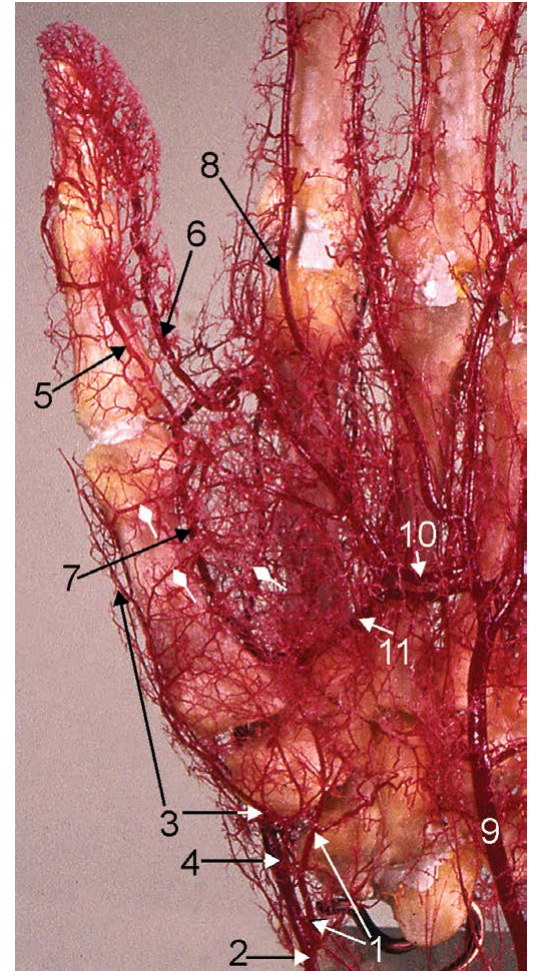

Figure 5. Ventral (palmar) view of the corrosion cast of the left hand with preserved bones. Note the hypoplastic superficial palmar branch (1) of the radial artery (2), the lateral thenar artery (3), and their anastomotic vessels (arrows), the middle thenar artery (4), the lateral palmar digital artery of the thumb (5), and the medial palmar digital artery of the thumb (6) that arise from the princeps pollicis artery (7); 8 - radialis indicis artery; 9 - ulnar artery; 10 - superficial palmar arch; 11 - deep palmar arch. 

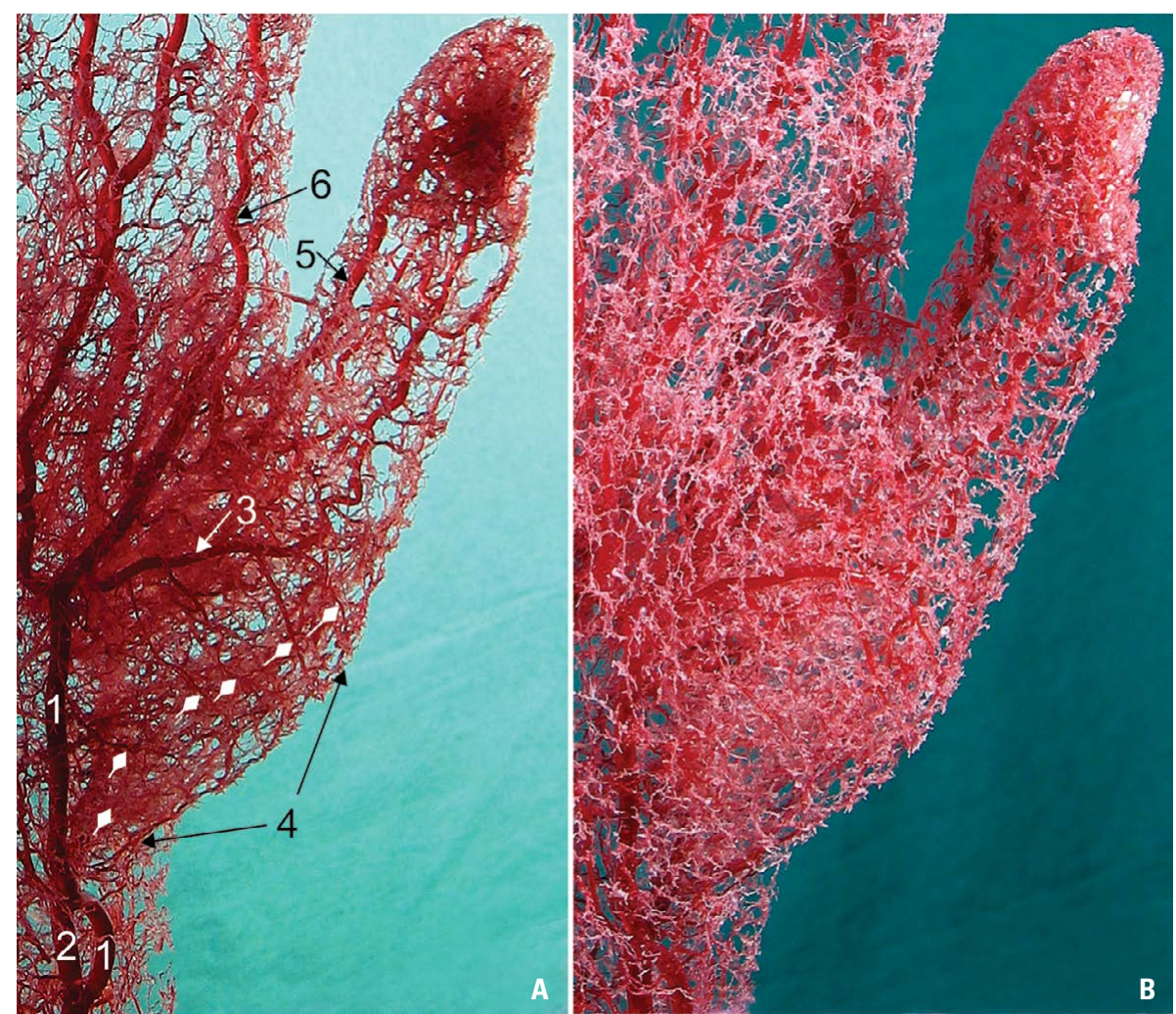

Figure 6. Long subcutaneous branches (arrows) originating from the prominent superficial palmar branch (1) of the radial artery (2), and the lateral palmar digital artery of the thumb (3), connected by anastomoses with the lateral thenar artery branches (4); A. Transillumination; 5 - medial palmar digital artery of the thumb; 6 - radialis indicis artery. They give off smaller, short star-like twigs to the skin of the thenar eminence; B. Side illumination, the same hand (ventral side of the vascular cast of the left hand).

subcutaneous tissue over the tuberosity of the scaphoid, in the majority of cases ( $80 \%$ ) over surface of the abductor pollicis brevis muscle, or along its deep side (20\%). SPB gave off a number of branches to the thenar muscles, and subcutaneously it also sent branches to the thenar skin. These continuous series of long and slender cutaneous branches had anastomotic connections with the LTA and MTA branches in the thenar fascia. The diameter of the SPB at its base on the RA ranged from 0.8 to $2.7 \mathrm{~mm}$ (mean $1.47 \mathrm{~mm}$ ).

We distinguished three types of SPB of the RA: prominent, average and hypoplastic, based on the significant variation in its diameter and vascularisation area.

The prominent SPB of the RA was defined as being connected to the superficial palmar arch of the ulnar artery by one vessel, sending cutaneous anastomotic branches to the thenar eminence skin, and continuing towards the index finger. The prominent SPB was found in $12(40 \%)$ specimens, and its diameter was over $1.70 \mathrm{~mm}$. Further, this branch supplied the lateral side of index finger (in all 12 hands), the medial side of the thumb (6 hands), or both sides of the thumb (3 hands) (Figs. 4A, B; 6).

The average SPB was also connected to the superficial palmar arch of the ulnar artery by one vessel; it anastomosed with the other arteries in the thenar fascia, and supplied the medial third of the thenar eminence (Figs. 1-3, 4C). This branch was found in $12(40 \%)$ specimens, and its diameter ranged from 1.0 to $1.7 \mathrm{~mm}$.

The hypoplastic SPB was found in $6(20 \%)$ specimens. This branch went into the proximal part of the thenar eminence fascia, where it ramified and had multiple small anastomoses with the superficial palmar arch of the ulnar artery, and the thenar arteries (Fig. 5). Its diameter ranged from 0.8 to $1.0 \mathrm{~mm}$.

We found the diameter of the SPB to be directly proportional to the thenar surface of the skin supplied by their branches, or to size of the cutaneous field of LTA and MTA. However, LTA and MTA were always present, even in the largest diameter of the SPB, and they irrigated constantly the skin of the lateral part of the thenar eminence. Long subcutaneous branches of 
these three arteries were interconnected via numerous anastomotic channels. They gave off a number of short twigs to the skin that pierced the thenar fascia surface, and rapidly divided in all directions in a star-like pattern (Figs. 2-6).

\section{DISCUSSION}

Today, it is a lack of more general study on the limb vasculature with embryological background [10]. The anatomists describe variations in the arterial blood perfusion pattern, found quite commonly during routine dissection, and surgeons apply this understanding of vascular territories in performing different kinds of flaps [6]. We added new information, and we completed an incomplete picture about the arterial supply of the thenar eminence skin. The largest part of the skin of the thenar eminence is supplied by SPB of the RA. This area includes the proximal part of the thenar eminence, over the abductor pollicis brevis muscle and the flexor pollicis brevis muscle $[4,13]$. Our results confirmed the observation that one can raise a fasciocutaneous flap from the thenar eminence based on the SPB, and transfer it as a free flap [13]. In all the specimens that we studied, SPB was connected to other palmar arteries by: a larger vessel in the prominent type, a smaller vessel in the average type, or by multiple small connections in the hypoplastic type of the SPB. In contrast to our finding, the literature mentions such connections of SPB to other palmar arteries in $63 \%$ of specimens [13]. Because of the always present distal anastomoses the afore mentioned flap could be transferred as a reverse-pedicled, with possible extension towards the LTA field of supply, in order to cover the larger defects of the palm, index finger, and thumb $[8,17]$. We have found the prominent SPB of the RA in 12 (40\%) specimens, with its diameter over $1.70 \mathrm{~mm}$, and dominantly supplying the skin of the thenar eminence, index finger (in all $40 \%$ of cases) and the thumb (in 30\% of cases). Omokawa et al. [13] reported that the SPB had an diameter larger than $2.00 \mathrm{~mm}$ in $17 \%$ of cases, provided the dominant supply to the thumb and index finger, and supplied the whole area of the thenar eminence. This and previous incidences were lower than one in the current study and might be a consequence of the different methodology. Our investigation is the first performed using a resin casts of the arteries obtained by corrosion of the injected human hands.
The microanatomical characteristics of the two branches of RA dorsal portion within the anatomical snuff-box seem to be almost overseen by other authors. The existence of the so-called thenar branches of the RA approaching from the dorsolateral side to the lateral and middle portions of the thenar should be respected during the microvascular surgery. However, in the literature we found similar descriptions of the LTA, and no data on the MTA. The same area has been studied previously by two authors [1, 12]. Bertelli et al. [1, 2] studied 'the first dorsal metacarpal artery' with clinical application as a donor site for a vascularised bone graft in scaphoid bone non-union. Moschella and Cordova [12] reported 'the dorsal radial digital collateral artery' following the radial side of the thumb, and they proposed the reverse pedicled skin flap on this artery as a useful option for repairing the thumb. According to the positions and branching patterns, arteries of both descriptions correspond to our LTA. Two clinical reports described use of a snuff-box flap, free or pedicled, designed to overlie the snuff-box, with its longer axis extending towards the ulnar side of the dorsum of the hand $[9,18]$. They confirmed that a few cutaneous perforators derived from the RA at the site of the anatomical snuff-box, but the inclination of the medial half of the flap towards the ulnar side of the hand dorsum is opposite to the situs and pattern of the thenar perforators. The longer axis of the flap followed the path of the first dorsal metacarpal artery. Another group of authors reported their experiences with application of the snuff-box cutaneous flap in 10 patients with skin contracture in the first web site and wrist [7]. The flap was harvested with a centre in the snuff-box region and bearing a width of 3-5 cm and a length of $10-14 \mathrm{~cm}$. This flap was based on the perforating branches from the RA in the snuff-box area, with no specific description of their microanatomical characteristics.

Based on our corrosion cast microanatomical study with detailed description of the LTA and MTA, the two regular branches of the RA in the space of the anatomical snuff-box, it is obvious that different fasciocutaneous flaps could be raised. Our finding that the LTA mean diameter was $0.67 \mathrm{~mm}$, comparing to the $1 \mathrm{~mm}$ on average [1], and that its cutaneous vascular territory over the lateral third of the thenar eminence was constant, suggests the possibility to proximally raise a fasciocutaneous flap based on LTA at its point of origin from RA. Area of skin that could be used as a flap supplied by LTA may be extended 
by its anastomotic channels with adjacent cutaneous branches of the SPB of the RA. The LTA flap could serve as a coverage of palmar and dorsal carpal defects. The free flap could be harvested from the lateral border of the thenar eminence with, or without the RA in its vascular pedicle (because of the size of the LTA), with its terminal part at the level of metacarpophalangeal joint of the thumb. The potential territory is thus larger than the anatomical vascular territory of the skin supplied by LTA branching pattern, and the vascular interconnections with SPB branches could allow expansion of the donor site for the thenar flap. These distal anastomoses could be able to feed a reverseflow distally based flap [12].

\section{CONCLUSIONS}

Three individually developed branches of the RA supply the skin of the thenar eminence. Cutaneous branches of these three arteries are interconnected via anastomotic vessels. We hope that our detailed anatomical descriptions will help in improving the surgical procedures that use a free or pedicled thenar flaps for coverage of palmar and dorsal skin defects.

\section{Acknowledgements}

This work was supported by grant No. 175030 from the Ministry of Education, Science and Technological Development of the Republic of Serbia.

\section{REFERENCES}

1. Bertelli JA, Pagliei A, Lassau JP. Role of the first dorsal metacarpal artery in the construction of pedicled bone grafts (27.3.92). Surg Radiol Anat. 1992; 14(3): 275-277, indexed in Pubmed: 1440194.

2. Bertelli JA, Tacca CP, Rost JR. Thumb metacarpal vascularized bone graft in long-standing scaphoid nonunion - a useful graft via dorsal or palmar approach: a cohort study of 24 patients. J Hand Surg Am. 2004; 29(6): 1089-1097, doi: 10.1016/j.jhsa.2004.06.007, indexed in Pubmed: 15576221.

3. Bilge $O$, Pinar $Y$, Ozer MA, et al. A morphometric study on the superficial palmar arch of the hand. Surg Radiol Anat. 2006; 28(4): 343-350, doi: 10.1007/s00276-006-0109-9, indexed in Pubmed: 16642281.

4. Cormack GC, Lamberty BGH. The arterial anatomy of skin flaps. Churchill Livingstone, Edinburgh-Tokyo 1994: 210-213.
5. Fitoussi $F$, Ghorbani $A$, Jehanno $P$, et al. Thenar flap for severe finger tip injuries in children. J Hand Surg Br. 2004; 29(2): 108-112, doi: 10.1016/j.jhsb.2003.10.006, indexed in Pubmed: 15010153.

6. Glotzbach JP, Levi B, Wong VW, et al. The basic science of vascular biology: implications for the practicing surgeon. Plast Reconstr Surg. 2010; 126(5): 1528-1538, doi: $10.1097 /$ PRS.0b013e3181 ef8ccf, indexed in Pubmed: 21042110.

7. Gu YD, Zhang GM, Lao J, et al. Application of snuffbox flaps based on branches from the radial artery. J Hand Surg. 1996; 21(6): 831-831, doi: 10.1016/s02667681(96)80207-5.

8. Hugon S, Castus P, Schoofs M. Index reconstruction by means of a fasciocutaneous thenar flap. Plast Reconstr Surg. 2010; 126(1): 43e-44e, doi: 10.1097/ PRS.0b013e3181 dab473, indexed in Pubmed: 20595849.

9. Inoue $T$, Ueda $K$, Kurihara $T$, et al. A new cutaneous flap: snuff-box flap. Br J Plast Surg. 1993; 46(3): 252-254, indexed in Pubmed: 8490707.

10. Klimek-Piotrowska W, Pacholczak R, Walocha J. Multiple variations of the arterial pattern in upper extremities: a case report and embryological pathogenesis. Clin Anat. 2013; 26(8): 1031-1035, doi: 10.1002/ca.22171, indexed in Pubmed: 23037918.

11. Loukas M, Holdman D, Holdman S. Anatomical variations of the superficial and deep palmar arches. Folia Morphol. 2005; 64(2): 78-83, indexed in Pubmed: 16121323.

12. Moschella F, Cordova A. Reverse homodigital dorsal radial flap of the thumb. Plast Reconstr Surg. 2006; 117(3): 920-926, doi: 10.1097/01.prs.0000200665.73624.6d, indexed in Pubmed: 16525286.

13. Omokawa S, Ryu J, Tang JB, et al. Vascular and neural anatomy of the thenar area of the hand: its surgical applications. Plast Reconstr Surg. 1997; 99(1): 116-121, indexed in Pubmed: 8982194.

14. Pistre V, Pelissier P, Martin D, et al. Vascular blood supply of the dorsal side of the thumb, first web and index finger: anatomical study. J Hand Surg Br. 2001; 26(2): 98-104, doi: 10.1054/jhsb.2000.0538, indexed in Pubmed: 11281658.

15. Ross AC. Wrist and Hand. In: Standring S (Editor-in-Chief), Gray's anatomy. The anatomical basis of clinical practice. Elsevier Limited, Philadelphia 2016: 862-897.

16. Salmon M. Arteries of the skin. Churchill Livingston, London-New York 1988: 32-34.

17. Seyhan T. Reverse thenar perforator flap for volar hand reconstruction. J Plast Reconstr Aesthet Surg. 2009; 62(10): 1309-1316, doi: 10.1016/j.bjps.2008.03.061, indexed in Pubmed: 18667372.

18. Takamatsu $A$, Inoue $T$, Kurihara $T$, et al. Free snuff-box flap for reconstruction of the wrap-around flap donor site. Br J Plast Surg. 1995; 48(5): 312-317, indexed in Pubmed: 7633769. 